Scenarios were identified using the top 13 organ involvement combinations, then patients were grouped into 7 categories based on GC dose and 10 patients per category were selected. Scenario information included: SLEDAI-2K score, organ involvement combination and GC dose.

3 rheumatologists ranked disease activity with PGA. An independent cohort was used for the validation in phase 3. We hypothesised that in patients with improvement/worsening by SLEDAI-2K, the change in SLEDAI-2K and SGI will correlate.

Results Scenario development is summarised in table 1. 131 scenarios were ranked by 3 rheumatologists leading to 393 records. Perfect LS agreement was achieved; ICC $(2, \mathrm{k})$ of 0.89 (95\% CI: $0.83,0.89)$. A quadratic linear regression model relating GC and SLEDAI-2K was structured; SGI score $=$ SLEDAI-2K $\quad$ score $+\left[3.65+0.29^{*} \mathrm{GC}-0.0027(\mathrm{GC} * \mathrm{GC})\right]$. The weight score of GC doses was derived (Table 2). Construct Validity: 109 of the 158 patients improved, 38 remained unchanged, 11 worsened. SLEDAI-2K and SGI correlated highly $(\mathrm{r}=0.87)$ and changed in the same direction in patients with improvement/worsening proving the validity of SGI.

Conclusions We developed and validated a novel lupus disease activity index, SGI, that describes disease activity while accounting for GC dose.

\section{VALIDATION OF A NOVEL DEFINITION OF LOW DISEASE ACTIVITY STATE IN SYSTEMIC LUPUS ERYTHEMATOSUS}

ALH Lee, T Cheung, HY Chung, CTK Ho, TWL Li, PH Li, MY Mok, H Tsang, RWS Wong, CKY Yuen, CS Lau*. The University of Hong Kong, Department of Medicine Division of Rheumatology and Clinical Immunology, Hong Kong, Hong Kong S.A.R

\subsection{6/upus-2017-000215.12}

Background and aims While a "Treat-to-target" principle is widely advocated in management of systemic lupus erythematosus (SLE), there is currently no internationally agreed definition of low disease activity in lupus. In 2015, the Asia-Pacific Lupus Collaboration presented a novel consensus definition of a safe state in lupus, the "Lupus Low Disease Activity State" (LLDAS). (http://dx.doi.org/10.1136/annrheumdis-2015-207726) Methods This was a prospective study to determine whether LLDAS predicted lower flare-ups, damage and mortality. 339 SLE patients were recruited and followed for 30 months. Multivariable binomial regression was used to determine factors associated with LLDAS and Cox proportional hazard model to determine whether prior higher\% of days in LLDAS would be associated with lower future flare-ups.

Results Mean patient age was 48.1 years and mean disease duration 19.6 years. Female to male ratio=16 to 1.79 flareups were documented. $92.6 \%$ of patients had ever achieved LLDAS during the study period and $62.1 \%$ of patient-days were in LLDAS. No major demographic or prior disease presentations were found to be associated with the attainment of LLDAS. Patients with prior higher percentage of days in LLDAS had lower hazard of lupus flare-ups $(H R=0.420$, $\mathrm{p}=0.015)$ after adjustment for gender and age. The numbers of patient damage and death were insufficient for analysis.

Conclusions LLDAS is an independent construct achievable by most patients of different history or background. Our preliminary study shows LLDAS can predict the risk of future flareups though further studies are needed to determine whether it is associated less lupus-related damage and lower mortality.

\section{Parallel Session 1: CNS lupus}

\section{ANTIBODY-MEDIATED NEUROPSYCHIATRIC LUPUS}

${ }^{1} \mathrm{~B}$ Diamond*, ${ }^{1} Y$ Arinuma, ${ }^{1} \mathrm{~F}$ Yuichiro, ${ }^{1} \mathrm{C}$ Kowal, ${ }^{1} \mathrm{~J}$ Nestor, ${ }^{2} \mathrm{P}$ Huerta, ${ }^{3} \mathrm{~B}$ Volpe. ${ }^{1}$ The Feinstein Insitute for Medical Research, Autoimmune and Musculoskeletal Diseases, Manhasset, USA; ${ }^{2}$ The Feinstein Institute for Medical Research, Biomedical Science, Manhasset, USA; ${ }^{3}$ The Feinstein Institute for Medical Research, Functional Neuroanatomy, Manhasset, USA

\subsection{6/lupus-2017-000215.13}

Neuropsychiatric lupus manifestations, especially the common disorders of mood and cognition, can be mediated by cytokines or by antibodies. In particular, a subset of anti-DNA antibodies has been shown to bind the $\mathrm{N}$-methyl d-aspartate receptor. The antibodies preferentially bind the active configuration of the receptor, augmenting the effects of ligand binding. Meta-analysis has confirmed that high serum titers of these antibodies, present in $30 \%-40 \%$ of SLE patients, are associated with cognitive impairment.

In a mouse model, antibody within the circulation is not harmful to the brain unless there is a breach in blood-brain barrier integrity. If there is an insult to the hippocampal vasculature, antibody gains access to hippocampal neurons and mediates tissue damage in 2 distinct stages. First, there is immediate antibody-mediated excitotoxicity causing neuronal loss. Second, after antibody is no longer present in the brain, there is microglial activation and dendritic pruning of surviving neurons. The brain injury that ensues leads to impairment in spatial cognition.

These studies suggest there are two distinct approaches to therapy; one might modulate the initial damage by neutralising the pathogenic antibodies or maintaining blood-brain barrier integrity, the other might mitigate the later damage by inhibiting microglial cells.

\section{INCREASED HETEROGENEITY OF BRAIN PERFUSION IS AN EARLY MARKER OF CENTRAL NERVOUS SYSTEM INVOLVEMENT IN ANTIPHOSPHOLIPID ANTIBODY CARRIERS}

${ }^{1} \mathrm{TS}$ Lin*, ${ }^{2} \mathrm{PY} \mathrm{Hsu},{ }^{3} \mathrm{CH}$ Chang, ${ }^{2} \mathrm{CL}$ Ko, ${ }^{4} \mathrm{SC}$ Hsieh. ${ }^{1}$ National Taiwan University Hospital Yun-Lin Branch, Internal Medicine, Yunlin, Taiwan R.O.C; ${ }^{2}$ National Taiwan University Hospital - Yun-Lin Branch, Nuclear Medicine, Yunlin, Taiwan R.O.C; ${ }^{3}$ National Taiwan University Hospital, Medical Research, Taipei, Taiwan R.O.C; ${ }^{4}$ National Taiwan University Hospital, Internal Medicine, Taipei, Taiwan R.O.C

\subsection{6/lupus-2017-000215.14}

Background and Aims The non-criteria neuropsychiatric manifestations of antiphospholipid syndrome include headache, dizziness, vertigo, seizure, depression and psychosis. There was still no objective method qualified to detect the early central nervous system involvement in non-criteria antiphospholipid syndrome. We evaluated the effectiveness of Tc-99m ECD SPECT in assessing circulatory insufficiency in the brains of patients with antiphospholipid antibodies and neuropsychiatric symptoms but without thromboembolism.

Methods Patients with a history of positive antiphospholipid antibodies and neuropsychiatric symptoms but without thromboembolism composed the case group; patients without antiphospholipid antibody served as the control group. Subjects with autoantibodies to extractable nuclear antigens were 